\title{
Algorithms \& Architectures at the Boundary of Signal Processing \& Machine Learning
}

\author{
John McAllister ${ }^{1}$
}

Accepted: 20 August 2021 / Published online: 1 October 2021

(c) Crown 2021

Signal processing and machine learning underpin a very wide spectrum of intelligent electronic devices, helping us locate, monitor, communicate with and control a wide variety of systems. Their increasing penetration into more extreme settings, at both large and small scales makes critical efficient design of algorithms and the devices on which they execute. These issues are only compounded by concerns of sustainability, waste and energy efficiency. This issue considers technologies which address this challenge, across the algorithm/architecture codesign spectrum.

An important factor in ensuring efficient implementation are the architectures used and their suitability to the task at hand. This issue presents a series of innovative architectures for operations key to modern signal processing systems. Machine learning, and particularly deep neural networks are a key emerging technology and the paper An Efficient FIFO based accelerator for Convolutional Neural Networks describes a circuit tuned to a specific, important type of modern neural network. Another type of application-communications, whether in wired or wireless forms-has long demanded highly efficient custom architectures. The paper Back-to-Back Butterfly, an Adaptive Permutation Network for New Communication Standards highlights how these can be expected to change with emerging communications standards, whilst the papers Exploring Energy Efficient Architectures for RLWE Lattice-Based Cryptography and Hardware Implementation for Bipartite Belief Propagation Polar Decoding with Bit Flipping offer new insights into important associated operations - reliable and secure lightweight data transport. Of course, the emergence of modern operations of this kind has prompted designers to re-evaluate approaches to more flexible programmable devices. The paper Floating Point CGRA based Ultra-Low Power DSP Accelerator is a good example of an architecture of this kind, whilst A Compute Cache System for Signal Processing Applications presents techniques for optimizing well-established architectural styles for signal processing applications.

Similarly, though, architectures can only provide most efficient operation if the algorithms they execute are inherently similar. A number of papers illustrate the potential for development of novel algorithms with this goal. The paper PureMIC: A New Audio Dataset for the Classification of Musical Instruments based on Convolutional Neural Networks presents optimised convolution neural networks for a novel kind of application. Efficient processing of audio, voice, images and video are not only long-standing domains requiring efficient system design, but remain areas of great challenges. The papers Real-time, Robust and Adaptive Universal Adversarial Attacks Against Speaker Recognition Systems studies how voice systems can be vulnerable to attack, whilst Real-Time Adaptive Multiple Transforms for the Next Generation Software Video Decoders and Object Detection in RGB-D Images via Anchor Box with Multi-Reduced Region Proposal Network and Multi-Pooling outline new avenues in image and video processing where standards are increasingly seeking new capabilities. Of course, the domain of application of signal processing technology is constantly growing as evidenced in the paper Forecasting Financial Time Series using Robust Deep Adaptive Input Normalization, which applies signal processing techniques in an area not usually the preserve of this kind of technology.

Publisher's Note Springer Nature remains neutral with regard to jurisdictional claims in published maps and institutional affiliations.
John McAllister

jp.mcallister@qub.ac.uk

1 Institute of Electronics. Communications and Information Technology (ECIT), Queen's University, Belfast, UK 